\title{
Sojourn Times in a Queueing System with Breakdowns and General Retrial Times
}

\author{
Ivan Atencia *,+ $\mathbb{D}$ and José Luis Galán-García ${ }^{+}(\mathbb{D}$ \\ Department of Applied Mathematics, University of Málaga, 29071 Málaga, Spain; jlgalan@uma.es \\ * Correspondence: iatencia@ctima.uma.es \\ + These authors contributed equally to this work.
}

Citation: Atencia, I.; Galán-García , J.L. Sojourn Times in a Queueing System with Breakdowns and General Retrial Times. Mathematics 2021, 9, 2882. https://doi.org/ $10.3390 /$ math 9222882

Academic Editor: Tuan Phung-Duc

Received: 13 October 2021

Accepted: 10 November 2021

Published: 12 November 2021

Publisher's Note: MDPI stays neutral with regard to jurisdictional claims in published maps and institutional affiliations.

\begin{abstract}
This paper centers on a discrete-time retrial queue where the server experiences breakdowns and repairs when arriving customers may opt to follow a discipline of a last-come, first-served (LCFS)type or to join the orbit. We focused on the extensive analysis of the system, and we obtained the stationary distributions of the number of customers in the orbit and in the system by applying the generation function (GF). We provide the stochastic decomposition law and the application bounds for the proximity between the steady-state distributions for the queueing system under consideration and its corresponding standard system. We developed recursive formulae aimed the calculation of the steady-state of the orbit and the system. We proved that our discrete-time system approximates $M / G / 1$ with breakdowns and repairs. We analyzed the busy period of an auxiliary system, the objective of which was to study the customer's delay. The stationary distribution of a customer's sojourn in the orbit and in the system was the object of a thorough and complete study. Finally, we provide numerical examples that outline the effect of the parameters on several performance characteristics and a conclusions section resuming the main research contributions of the paper.
\end{abstract}

Keywords: discrete-time queueing system; general retrial times; breakdown; repairs; sojourn times

\section{Introduction}

Queueing systems are an important tool in different applications such as performance analysis and modeling. These types of systems are in our everyday life activities, and the theory of queueing systems was developed to provide models for forecasting the behaviors of systems subject to random demand. The practical and useful applications of discrete-time queues motivate researchers to continue investigating these models.

Service disruptions are an inescapable occurrence in a wide variety of real-world circumstances. In actuality, we often encounter a situation when a server fails and can be fixed. The applications of such models can be found in the areas of computer communication networks and flexible manufacturing systems. For a general survey on service disruptions, we refer to [1-4].

Numerous queueing systems may serve tasks repeatedly for a variety of reasons. When a job is not performed satisfactorily, the task may be repeated as many times as required until the task is completed successfully. This kind of queueing system arises in the stochastic modeling of a wide variety of real-world scenarios. For example, in data transmission, a packet transmitted from the source to the destination can be returned or it can continue until the packet is finally transmitted. In the retrial group, upon the completion of each service, the server will remain idle in the system until the arrival of the next primary or retrial task.

A retrial queueing model is characterized by the fact that when an arriving customer or task encounters a busy server, it is displaced or expelled by the system, as illustrated in $[5,6]$, and thus neither joins the waiting line nor exits the system immediately, but instead, travels to some virtual space, called the orbit, and retries obtaining service after 
some random time. For a study of this subject, see [1]; for engineering applications, see [7]; for communication network applications, see $[8,9]$.

Such queueing systems arise in many communication protocols, local area networks, wireless and computer networks, and everyday life situations. For a general survey on retrial queues and a summary of many results, we refer to [10-12].

The rest of this work is organized in the following manner. The next section discusses the assumptions of the queueing system. Section 3 is devoted to the Markov chain. The queue and system size distributions are determined in conjunction with numerous system performance measures. Section 4 presents a stochastic decomposition property and, as an application, upper and lower bounds on the distance between the studied system's steadystate distribution and its corresponding standard system. In Section 5, we devise recursive formulas to determine the steady-state distributions of the orbit and system sizes. Section 6 examines the relationship between continuous-time and discrete-time systems. In Section 7, the busy period of an auxiliary system is determined, which is valuable for analyzing customer delay. In Section 8, we detail the sojourn periods of a customer in the queue and across the system, along with their associated methods. Section 9 contains numerical findings illustrating the influence of the settings on a variety of performance factors. Finally, a conclusions section is offered that discusses the paper's primary scientific contributions.

\section{The Mathematical Model}

In this section, we present the mathematical model that was used in this work. Specifically, we considered a discrete-time queueing system where the time axis is split into a sequence of equal intervals, $0,1, \ldots, m, \ldots$, called slots. We assumed that arrivals, departures, retrials, and repairs (all queueing activities) take place around the slot boundaries. We considered the epoch $m$ and supposed that the departure and ending of repairs occur in the interval $\left(\mathrm{m}^{-}, \mathrm{m}\right)$ and the arrivals, beginning of repairs, and retrials occur in the interval $\left(m, m^{+}\right)$, in this order.

The stream of incoming customers into the system is described by means of a Bernoulli process where $a$ is the probability that an arrival occurs in a slot. If an arriving customer finds the server idle, it begins its service immediately; otherwise, the following two situations can occur: (1) with probability $\theta$, the arriving customer is expels from the system the customer that is currently being served and begins its service or (2) with complementary probability $\bar{\theta}$, it enters a group of blocked customers called the "orbit" in accordance with a first-come, first-served (FCFS) discipline. We assumed that only the customer at the head of the orbit is allowed access to the server. During a service time, failures in the server may occur, and in this case, the customer that was taking its service is placed at the head of the orbit. Customers that enter the system when the server is down go directly to the orbit. It was assumed that the server lifetime is geometrically distributed with parameter $v=1-\bar{v}$, where $v$ is the probability that a failure does not occur in a slot.

Successive inter-retrial times are governed by an arbitrary distribution $\left\{a_{i}\right\}_{i=0}^{\infty}$ with generating function $A(x)=\sum_{i=0}^{\infty} a_{i} x^{i}$. The service times are independent and distributed with arbitrary distribution $\left\{s_{i}\right\}_{i=1}^{\infty}$ and generating function $S(x)=\sum_{i=1}^{\infty} s_{i} x^{i}$. Hence, $s_{i}$ is the probability that a service lasts $i$ slots. The probability that the service times last no less than $k$ slots is denoted by $S_{k}=\sum_{i=k}^{\infty} s_{i}$. The repair times are governed by an arbitrary distribution $\left\{r_{i}\right\}_{i=1}^{\infty}$ with generating function $R(x)=\sum_{i=1}^{\infty} r_{i} x^{i}$ 


\section{The Markov Chain}

At time $m^{+}$, the state of the system can be described by the process:

$$
Y_{m}=\left(C_{m}, \xi_{0, m}, \xi_{1, m}, \xi_{2, m}, N_{m}\right)
$$

where $C_{m}$ denotes the state of the server, 0,1 , or 2 according to whether the server is free, busy, or under repair, and $N_{m}$ is the number of repeat customers: iff $C_{m}=0$ and $N_{m}>0, \xi_{0, m}$ represents the remaining retrial time; if $C_{m}=1,2 \xi_{1, m}$ and $\xi_{2, m}$ correspond to the remaining service and repair time, respectively.

It can be shown that $\left\{Y_{m}: m \in \mathbb{N}\right\}$ is the Markov chain of the queueing system, whose states space is:

$$
\{(0,0) ;(0, i, k): i \geq 1, k \geq 1 ;(1, i, k): i \geq 1, k \geq 0 ;(2, i, k): i \geq 1, k \geq 1\} .
$$

Our objective is to find the stationary distribution:

$$
\begin{aligned}
& \pi_{0,0}=\lim _{m \rightarrow \infty} P\left[C_{m}=0, N_{m}=0\right] \\
& \pi_{0, i, k}=\lim _{m \rightarrow \infty} P\left[C_{m}=0, \xi_{0, m}=i, N_{m}=k\right] ; i \geq 1, k \geq 1, \\
& \pi_{1, i, k}=\lim _{m \rightarrow \infty} P\left[C_{m}=1, \xi_{1, m}=i, N_{m}=k\right] ; i \geq 1, k \geq 0, \\
& \pi_{2, i, k}=\lim _{m \rightarrow \infty} P\left[C_{m}=2, \xi_{2, m}=i, N_{m}=k\right] ; i \geq 1, k \geq 1,
\end{aligned}
$$

of the Markov chain $\left\{Y_{m}: m \in \mathbb{N}\right\}$.

The system of equilibrium equations (SEE) for the stationary distribution is:

$$
\begin{aligned}
\pi_{0,0} & =\bar{a} \pi_{0,0}+\bar{a} \pi_{1,1,0} \Longleftrightarrow a \pi_{0,0}=\bar{a} \pi_{1,1,0} \\
\pi_{0, i, k} & =\bar{a} \pi_{0, i+1, k}+\bar{a} a_{i} \pi_{1,1, k}+\bar{a} a_{i} \pi_{2,1, k} ; i \geq 1, k \geq 1, \\
\pi_{1, i, k} & =\delta_{0, k} a s_{i} \pi_{0,0}+\bar{a} s_{i} \pi_{0,1, k+1}+\left(1-\delta_{0, k}\right) a s_{i} \sum_{j=1}^{\infty} \pi_{0, j, k}+ \\
& +a s_{i} \pi_{1,1, k}+\bar{a} a_{0} s_{i} \pi_{1,1, k+1}+ \\
& +\left(1-\delta_{0, k}\right) a \bar{\theta} v \pi_{1, i+1, k-1}+\bar{a} v \pi_{1, i+1, k}+ \\
& +a \theta s_{i} \sum_{j=2}^{\infty} \pi_{1, j, k}+ \\
& +\left(1-\delta_{0, k}\right) a s_{i} \pi_{2,1, k}+\bar{a} a_{0} s_{i} \pi_{2,1, k+1}, i \geq 1, k \geq 0 \\
\pi_{2, i, k} & =\left(1-\delta_{1, k}\right) a \pi_{2, i+1, k-1}+\bar{a} \pi_{2, i+1, k}+ \\
& +\left(1-\delta_{1, k}\right) a \bar{\theta} \bar{v} r_{i} \sum_{j=2}^{\infty} \pi_{1, j, k-2}+ \\
& +\bar{a} \bar{v} r_{i} \sum_{j=2}^{\infty} \pi_{1, j, k-1} ; i \geq 1, k \geq 1,
\end{aligned}
$$

where $\bar{a}=1-a$ and $\delta_{i, j}$ denotes Kronecker's delta.

The normalization condition is:

$$
\pi_{0,0}+\sum_{i=1}^{\infty} \sum_{k=1}^{\infty} \pi_{0, i, k}+\sum_{i=1}^{\infty} \sum_{k=0}^{\infty} \pi_{1, i, k}+\sum_{i=1}^{\infty} \sum_{k=1}^{\infty} \pi_{2, i, k}=1 .
$$


To solve Equations (1)-(4), we use the following generating functions:

$$
\begin{aligned}
& \varphi_{0}(x, z)=\sum_{i=1}^{\infty} \sum_{k=1}^{\infty} \pi_{0, i, k} x^{i} z^{k} \\
& \varphi_{1}(x, z)=\sum_{i=1}^{\infty} \sum_{k=0}^{\infty} \pi_{1, i, k} x^{i} z^{k} \\
& \varphi_{2}(x, z)=\sum_{i=1}^{\infty} \sum_{k=1}^{\infty} \pi_{2, i, k} x^{i} z^{k},
\end{aligned}
$$

and the auxiliary generating functions:

$$
\begin{aligned}
\varphi_{0, i}(z) & =\sum_{k=1}^{\infty} \pi_{0, i, k} z^{k}, i \geq 1 \\
\varphi_{1, i}(z) & =\sum_{k=0}^{\infty} \pi_{1, i, k} z^{k}, i \geq 1 \\
\varphi_{2}(x, z) & =\sum_{k=1}^{\infty} \pi_{2, i, k} z^{k}, i \geq 1 .
\end{aligned}
$$

Multiplying (2)-(4) by $z^{k}$, summing over $k$, and taking into account (1), these equations become:

$$
\begin{aligned}
\varphi_{0, i}(z) & =\bar{a} \varphi_{0, i+1}(z)+\bar{a} a_{i} \varphi_{1,1}(z)+\bar{a} a_{i} \varphi_{2,1}(z)-a a_{i} \pi_{0,0}, i \geq 1 \\
\varphi_{1, i}(z) & =(\bar{a}+a \bar{\theta} z) v \varphi_{1, i+1}(z)+\frac{\bar{a}}{z} s_{i} \varphi_{0,1}(z)+a s_{i} \varphi_{0}(1, z)+ \\
& +\frac{\bar{a} a_{0}+a \bar{\theta} z}{z} s_{i} \varphi_{1,1}(z)+a \theta s_{i} \varphi_{1}(1, z)+ \\
& +\frac{\bar{a} a_{0}+a z}{z} s_{1} \varphi_{2,1}(z)+\frac{z-a_{0}}{z} a s_{i} \pi_{0,0}, i \geq 1, \\
\varphi_{2, i}(z) & =(\bar{a}+a z) \varphi_{2, i+1}(z)+(\bar{a}+a \bar{\theta} z) z \bar{v} r_{i} \varphi_{1}(1, z)- \\
& -(\bar{a}+a \bar{\theta} z) z \bar{v} r_{i} \varphi_{1,1}(z), i \geq 1,
\end{aligned}
$$

Then, multiplying (5)-(7) by $x^{i}$ and summing over $i$ lead to:

$$
\begin{aligned}
\frac{x-\bar{a}}{x} \varphi_{0}(x, z) & =\bar{a}\left[A(x)-a_{0}\right] \varphi_{1,1}(z)+\bar{a}\left[A(x)-a_{0}\right] \varphi_{2,1}(z)- \\
& -\bar{a} \varphi_{0,1}(z)-a\left[A(x)-a_{0}\right] \pi_{0,0}, \\
z \frac{x-(\bar{a}+a \bar{\theta} z) v}{x} \varphi_{1}(x, z) & =\bar{a} S(x) \varphi_{0,1}(z)+a z S(x) \varphi_{0}(1, z)+ \\
& +\left[\left(\bar{a} a_{0}+a \bar{\theta} z\right) S(x)-z(\bar{a}+a \bar{\theta} z) v\right] \varphi_{1,1}(z)+ \\
& +a \theta z S(x) \varphi_{1}(1, z)+\left(\bar{a} a_{0}+a z\right) S(x) \varphi_{2,1}(z)+ \\
& +\left(z-a_{0}\right) a S(x) \pi_{0,0} \\
\frac{x-(\bar{a}+a z)}{x} \varphi_{2}(x, z) & =(\bar{a}+a \bar{\theta} z) z \bar{v} R(x) \varphi_{1}(1, z)- \\
& -(\bar{a}+a \bar{\theta} z) z \bar{v} R(x) \varphi_{1,1}(z)-(\bar{a}+a z) \varphi_{2,1}(z),
\end{aligned}
$$


Choosing $x=1$ in (8) yields:

$$
\begin{aligned}
a \varphi_{0}(1, z) & =\bar{a}\left(1-a_{0}\right) \varphi_{1,1}(z)+\bar{a}\left(1-a_{0}\right) \varphi_{2,1}(z)-\bar{a} \varphi_{0,1}(z)- \\
& -a\left(1-a_{0}\right) \pi_{0,0}
\end{aligned}
$$

and inserting (11) in (9) gives:

$$
\begin{aligned}
z \frac{x-(\bar{a}+a \bar{\theta} z) v}{x} \varphi_{1}(x, z) & =\bar{a}(1-z) S(x) \varphi_{0,1}(z)+ \\
& +\left[\left(\bar{a} a_{0}(1-z)+(\bar{a}+a \bar{\theta}) z\right) S(x)-\right. \\
& -z(\bar{a}+a \bar{\theta} z) v] \varphi_{1,1}(z)+a \theta z S(x) \varphi_{1}(1, z)+ \\
& +\left[z+\bar{a} a_{0}(1-z)\right] S(x) \varphi_{2,1}(z)- \\
& -a a_{0}(1-z) S(x) \pi_{0,0}
\end{aligned}
$$

while setting $x=1$ in (12) yields:

$$
\begin{aligned}
z k(z) \varphi_{1}(1, z) & =\bar{a}(1-z) \varphi_{0,1}(z)+\left[\bar{a} a_{0}(1-z)+z k(z)\right] \varphi_{1,1}(z)+ \\
& +\left[z+\bar{a} a_{0}(1-z)\right] \varphi_{2,1}(z)-a a_{0}(1-z) \pi_{0,0} .
\end{aligned}
$$

where $k(z)=1-a \theta-(\bar{a}+a \bar{\theta} z) v$.

From (10), (12), and (13), we obtain:

$$
\begin{aligned}
z k(z) \frac{x-(\bar{a}+a \bar{\theta} z) v}{x} \varphi_{1}(x, z) & =\bar{a}(1-z)[1-(\bar{a}+a \bar{\theta} z) v] S(x) \varphi_{0,1}(z)+ \\
& +\left[\left(z k(z)+\bar{a} a_{0}(1-z)[1-(\bar{a}+a \bar{\theta} z) v]\right) S(x)-\right. \\
& -z k(z)(\bar{a}+a \bar{\theta} z) v] \varphi_{1,1}(z)+ \\
& +\left[z+\bar{a} a_{0}(1-z)\right][1-(\bar{a}+a \bar{\theta} z) v] S(x) \varphi_{2,1}(z)- \\
& -a a_{0}(1-z)[1-(\bar{a}+a \bar{\theta} z) v] S(x) \pi_{0,0} \\
k(z) \frac{x-(\bar{a}+a z)}{x} \varphi_{2}(x, z) & =\bar{a}(1-z)(\bar{a}+a \bar{\theta} z) \bar{v} R(x) \varphi_{0,1}(z)+ \\
& +\bar{a} a_{0}(1-z)(\bar{a}+a \bar{\theta} z) \bar{v} R(x) \varphi_{1,1}(z)+ \\
& +\left[\left[z+\bar{a} a_{0}(1-z)\right](\bar{a}+a \bar{\theta} z) \bar{v} R(x)-\right. \\
& -(\bar{a}+a z) k(z)] \varphi_{2,1}(z)- \\
& -a a_{0}(1-z)(\bar{a}+a \bar{\theta} z) \bar{v} R(x) \pi_{0,0}
\end{aligned}
$$

Setting $x=\bar{a}$ in (8), $x=(\bar{a}+a \bar{\theta} z) v$ in (14), and $x=\bar{a}+a z$ in (15), we obtain: 


$$
\begin{aligned}
& a\left[A(\bar{a})-a_{0}\right] \pi_{0,0}=\bar{a}\left[A(\bar{a})-a_{0}\right] \varphi_{1,1}(z)+\bar{a}\left[A(\bar{a})-a_{0}\right] \varphi_{2,1}(z)-\bar{a} \varphi_{0,1}(z) \\
& a a_{0}(1-z)[1-(\bar{a}+a \bar{\theta} z) v] S[(\bar{a}+a \bar{\theta} z) v] \pi_{0,0}= \\
= & \bar{a}(1-z)[1-(\bar{a}+a \bar{\theta} z) v] S[(\bar{a}+a \bar{\theta} z) v] \varphi_{0,1}(z)+ \\
+ & {\left[\left(z k(z)+\bar{a} a_{0}[1-z][1-(\bar{a}+a \bar{\theta} z) v]\right) S[(\bar{a}+a \bar{\theta} z) v]-\right.} \\
- & z k(z)(\bar{a}+a \bar{\theta} z) v] \varphi_{1,1}(z)+[z+ \\
+ & \left.\bar{a} a_{0}(1-z)\right][1-(\bar{a}+a \bar{\theta} z) v] S[(\bar{a}+a \bar{\theta} z) v] \varphi_{2,1}(z), \\
& a a_{0}(1-z)(\bar{a}+a \bar{\theta} z) \bar{v} R(\bar{a}+a z) \pi_{0,0}= \\
= & \bar{a}(1-z)(\bar{a}+a \bar{\theta} z) \bar{v} R(\bar{a}+a z) \varphi_{0,1}(z)+ \\
+ & \bar{a} a_{0}(1-z)(\bar{a}+a \bar{\theta} z) \bar{v} R(\bar{a}+a z) \varphi_{1,1}(z)+ \\
+ & {\left[\left[z+\bar{a} a_{0}(1-z)\right](\bar{a}+a \bar{\theta} z) \bar{v} R(\bar{a}+a z)-\right.} \\
- & (\bar{a}+a z) k(z)] \varphi_{2,1}(z),
\end{aligned}
$$

and from the above system, we obtain the generating functions $\varphi_{0,1}(z), \varphi_{1,1}(z)$, and $\varphi_{2,1}(z)$ :

$$
\begin{aligned}
\varphi_{0,1}(z)= & \frac{1}{D(z)}\left[a z\left[A(\bar{a})-a_{0}\right][(\bar{a}+a \bar{\theta} z) v-S[(\bar{a}+a \bar{\theta} z) v]] .\right. \\
\cdot & {[(\bar{a}+a z) k(z)-z(\bar{a}+a \bar{\theta} z) \bar{v} R(\bar{a}+a z)]] \frac{\pi_{0,0}}{\bar{a}} } \\
\varphi_{1,1}(z)= & \frac{a A(\bar{a})(1-z)[1-(\bar{a}+a \bar{\theta} z) v](\bar{a}+a z) S[(\bar{a}+a \bar{\theta} z) v]}{D(z)} \pi_{0,0} \\
\varphi_{2,1}(z)= & \frac{1}{D(z)}[a z A(\bar{a})(1-z)(\bar{a}+a \bar{\theta} z) \bar{v} R(\bar{a}+a z) . \\
\cdot & {[(\bar{a}+a \bar{\theta} z) v-S[(\bar{a}+a \bar{\theta} z) v]]] \pi_{0,0} }
\end{aligned}
$$

where:

$$
\begin{aligned}
D(z) & =\bar{a} A(\bar{a})(1-z)(\bar{a}+a z)[1-(\bar{a}+a \bar{\theta} z) v] S[(\bar{a}+a \bar{\theta} z) v]- \\
& -z[(\bar{a}+a \bar{\theta} z) v-S[(\bar{a}+a \bar{\theta} z) v]]\{(\bar{a}+a z) k(z)- \\
& -[z+\bar{a} A(\bar{a})(1-z)](\bar{a}+a \bar{\theta} z) \bar{v} R(\bar{a}+a z)\} .
\end{aligned}
$$

Substituting (16), (17), and (18) into (8), (14), and (15), we have the generating functions:

$$
\begin{aligned}
\varphi_{0}(x, z)= & \frac{A(x)-A(\bar{a})}{x-\bar{a}} . \\
& \cdot \frac{a x z[(\bar{a}+a \bar{\theta} z) v-S[(\bar{a}+a \bar{\theta} z) v]][(\bar{a}+a z) k(z)-z(\bar{a}+a \bar{\theta} z) \bar{v} R(\bar{a}+a z)]}{D(z)} \pi_{0,0}
\end{aligned}
$$




$$
\begin{aligned}
\varphi_{1}(x, z) & =\frac{S(x)-S[(\bar{a}+a \bar{\theta} z) v]}{x-(\bar{a}+a \bar{\theta} z) v} . \\
& \cdot \frac{a x A(\bar{a})(1-z)[1-(\bar{a}+a \bar{\theta} z) v](\bar{a}+a \bar{\theta} z) v(\bar{a}+a z)}{D(z)} \pi_{0,0} \\
\varphi_{2}(x, z)= & \frac{R(x)-R(\bar{a}+a z)}{x-(\bar{a}+a z)} \cdot \\
& \cdot \frac{a x z A(\bar{a})(1-z)(\bar{a}+a \bar{\theta} z) \bar{v}(\bar{a}+a z)[(\bar{a}+a \bar{\theta} z) v-S[(\bar{a}+a \bar{\theta} z) v]]}{D(z)} \pi_{0,0} .
\end{aligned}
$$

Using the normalization condition, which can be written as $\pi_{0,0}+\varphi_{0}(1,1)+\varphi_{1}(1,1)+$ $\varphi_{2}(1,1)=1$, we can find the unknown constant $\pi_{0,0}$ :

$$
\pi_{0,0}=\frac{-D^{\prime}(1)}{(\bar{a}+a \bar{\theta})[a \theta v+\bar{v} S[(\bar{a}+a \bar{\theta}) v]] A(\bar{a})}
$$

where:

$$
\begin{aligned}
-D^{\prime}(1) & =\bar{a} A(\bar{a})[1-(\bar{a}+a \bar{\theta}) v] S[(\bar{a}+a \bar{\theta}) v]- \\
& -[(\bar{a}+a \bar{\theta}) v-S[(\bar{a}+a \bar{\theta}) v]][a \bar{\theta}+ \\
& \left.+(\bar{a}+a \bar{\theta}) \bar{v}\left[\bar{a}[1-A(\bar{a})]+a R^{\prime}(1)\right]\right]
\end{aligned}
$$

We suppose that the condition:

$$
-D^{\prime}(1)>0
$$

is fulfilled in the rest of the paper.

We summarize the above results in the following theorem.

Theorem 1. The generating functions of the stationary distribution of the Markov chain $\left\{Y_{m}: m \in \mathbb{N}\right\}$ are given by:

$$
\begin{aligned}
\varphi_{0}(x, z)= & \frac{A(x)-A(\bar{a})}{x-\bar{a}} \cdot \\
& \cdot \frac{a x z[(\bar{a}+a \bar{\theta} z) v-S[(\bar{a}+a \bar{\theta} z) v]][(\bar{a}+a z) k(z)-z(\bar{a}+a \bar{\theta} z) \bar{v} R(\bar{a}+a z)]}{D(z)} \pi_{0,0} \\
\varphi_{1}(x, z)= & \frac{S(x)-S[(\bar{a}+a \bar{\theta} z) v]}{x-(\bar{a}+a \bar{\theta} z) v} \cdot \\
& \cdot \frac{a x A(\bar{a})(1-z)[1-(\bar{a}+a \bar{\theta} z) v](\bar{a}+a \bar{\theta} z) v(\bar{a}+a z)}{D(z)} \pi_{0,0} \\
\varphi_{2}(x, z)= & \frac{R(x)-R(\bar{a}+a z)}{x-(\bar{a}+a z)} \cdot \\
& \cdot \frac{a x z A(\bar{a})(1-z)(\bar{a}+a \bar{\theta} z) \bar{v}(\bar{a}+a z)[(\bar{a}+a \bar{\theta} z) v-S[(\bar{a}+a \bar{\theta} z) v]]}{D(z)} \pi_{0,0}
\end{aligned}
$$


where:

$$
\begin{aligned}
D(z) & =\bar{a} A(\bar{a})(1-z)(\bar{a}+a z)[1-(\bar{a}+a \bar{\theta} z) v] S[(\bar{a}+a \bar{\theta} z) v]- \\
& -z[(\bar{a}+a \bar{\theta} z) v-S[(\bar{a}+a \bar{\theta} z) v]]\{(\bar{a}+a z) k(z)- \\
& -[z+\bar{a} A(\bar{a})(1-z)](\bar{a}+a \bar{\theta} z) \bar{v} R(\bar{a}+a z)\} . \\
\pi_{0,0} & =\frac{-D^{\prime}(1)}{(\bar{a}+a \bar{\theta})[a \theta v+\bar{v} S[(\bar{a}+a \bar{\theta}) v]] A(\bar{a})}
\end{aligned}
$$

and:

$$
\begin{aligned}
D^{\prime}(1) & =\bar{a} A(\bar{a})[1-(\bar{a}+a \bar{\theta}) v] S[(\bar{a}+a \bar{\theta}) v]- \\
& -[(\bar{a}+a \bar{\theta}) v-S[(\bar{a}+a \bar{\theta}) v]]\left[a \bar{\theta}+(\bar{a}+a \bar{\theta}) \bar{v}\left[\bar{a}[1-A(\bar{a})]+a R^{\prime}(1)\right]\right]
\end{aligned}
$$

Corollary 1. 1. The probability-generating function of the number of customers in the retrial group (i.e., of the variable $N$ ) is given by:

$$
\begin{aligned}
\psi(z) & =\pi_{0,0}+\varphi_{0}(1, z)+\varphi_{1}(1, z)+\varphi_{2}(1, z)= \\
& =\frac{(1-z)(\bar{a}+a z)(\bar{a}+a \bar{\theta} z)[a(1-\bar{\theta} z) v+\bar{v} S[(\bar{a}+a \bar{\theta} z) v]]}{D(z)} A(\bar{a}) \pi_{0,0}
\end{aligned}
$$

2. The probability-generating function of the number of customers in the system (i.e., of the variable $L$ ) is given by:

$$
\begin{aligned}
\Phi(z) & =\pi_{0,0}+\varphi_{0}(1, z)+z \varphi_{1}(1, z)+\varphi_{2}(1, z)= \\
& =\frac{(1-z)(\bar{a}+a z)(\bar{a}+a \bar{\theta} z)[a \theta v z+[1-(\bar{a}+a z) v] S[(\bar{a}+a \bar{\theta} z) v]]}{D(z)} A(\bar{a}) \pi_{0,0}
\end{aligned}
$$

Corollary 2. 1. The mean number of customers in the retrial group is given by:

$$
E[N]=\psi^{\prime}(1)=\frac{\nabla}{\triangle}
$$

where:

$$
\begin{aligned}
\nabla & \left.=2 a D^{\prime}(1)\left\{[a \theta v+\bar{v}](\bar{a}+(1+a) \bar{\theta})-\bar{\theta} v(\bar{a}+a \bar{\theta})\left[1-\bar{v} S^{\prime}[(\bar{a}+a \bar{\theta}) v)\right]\right]\right\} \\
& -(\bar{a}+a \bar{\theta})[a \theta v+\bar{v} S[(\bar{a}+a \bar{\theta}) v]] D^{\prime \prime}(1)
\end{aligned}
$$

and:

$$
\triangle=2 D^{\prime}(1)(\bar{a}+a \bar{\theta})[a \theta v+\bar{v} S[(\bar{a}+a \bar{\theta}) v]
$$

with:

$$
\begin{aligned}
\frac{D^{\prime \prime}(1)}{2} & =-a \bar{a} A(\bar{a})[1-(\bar{a}+a \bar{\theta}) v]\left[S[(\bar{a}+a \bar{\theta}) v]+a \bar{\theta} v S^{\prime}[(\bar{a}+a \bar{\theta}) v]\right]+ \\
& +\left[\bar{a}+2 a \bar{\theta} v-S\left[(\bar{a}+a \bar{\theta}) v-a \bar{\theta} v S^{\prime}[(\bar{a}+a \bar{\theta}) v]\right][a+(\bar{a}+\right. \\
& \left.+a \bar{\theta}) \bar{v}\left[\bar{a}[1-A(\bar{a})]+a R^{\prime}(1)\right]\right]+a[(\bar{a}+a \bar{\theta}) v-S[(\bar{a}+a \bar{\theta}) v]][a(1-\bar{\theta} \bar{v})+ \\
& \left.+[1-\bar{a} A(\bar{a})]\left(\bar{\theta} v+(\bar{a}+2 a \bar{\theta} \bar{v}) R^{\prime}(1)\right)+\frac{1}{2}(\bar{a}+a \bar{\theta}) \bar{v} a^{2} R^{\prime \prime}(1)\right]
\end{aligned}
$$


2. The mean number of customers in the system is:

$$
E[L]=\Phi^{\prime}(1)=E[N]+\varphi_{1}(1,1),
$$

where:

$$
\varphi_{1}(1,1)=\frac{1-S[(\bar{a}+a \bar{\theta}) v]}{a \theta v+\bar{v} S[(\bar{a}+a \bar{\theta}) v]} a v .
$$

\section{Stochastic Decomposition}

Queueing systems with server vacations are studied using the stochastic decomposition law; see, for example, [13]. The above-mentioned law defines the distribution of the number of customers in the system as the sum of two independent random variables. One of them is the number of customers in the corresponding standard queueing system, which is the system without vacations. The second variable is the size of the system when the server is on vacation.

In [14,15], the stochastic decomposition in some discrete-time queueing systems was studied. This decomposition has also been analyzed for discrete-time Geo/G/1 retrial queues; see [5,16-18]. The system under study can be considered as a standard queue Geo $/ G / 1 / \infty$ with server breakdowns, repairs, and server vacations. This model describes a server that starts its vacation when a previous service finishes and there are no arrivals or retrials. The duration of the vacation depends on the arrival process and inter-retrial times. The vacation ends whenever an activity in the system is recognized, that is if an external customer arrives or a repeat customer retries for service.

The probability-generating function $\Phi(z)$ of the number of customers in the system can be written as:

$$
\Phi(z)=\Phi^{*}(z) \cdot \frac{\pi_{0,0}+\varphi_{0}(1, z)}{\pi_{0,0}+\varphi_{0}(1,1)}
$$

where the first factor $\Phi^{*}(z)$ is:

$$
\Phi^{*}(z)=\frac{(1-z)(\bar{a}+a \bar{\theta} z)[a \theta v z+[1-(\bar{a}+a z) v] S[(\bar{a}+a \bar{\theta} z) v]]}{\bar{a}(1-z)[1-(\bar{a}+a \bar{\theta} z) v] S[(\bar{a}+a \bar{\theta} z) v]-z[(\bar{a}+a \bar{\theta} z) v-S[(\bar{a}+a \bar{\theta} z) v]](k(z)-(\bar{a}+a \bar{\theta} z) \bar{v} R(\bar{a}+a z))} \cdot \pi_{0}^{*}
$$

with:

$$
\pi_{0}^{*}=\frac{\bar{a}(\bar{v}+a \theta v) S[(\bar{a}+a \bar{\theta}) v]-[(\bar{a}+a \bar{\theta}) v-S[(\bar{a}+a \bar{\theta}) v]]\left(a \bar{\theta}+(\bar{a}+a \bar{\theta}) \bar{v} a R^{\prime}(1)\right)}{(\bar{a}+a \bar{\theta})[a \theta v+\bar{v} S[(\bar{a}+a \bar{\theta}) v]]}
$$

corresponding to the probability-generating function of the number of customers in the queueing system Geo/G/1/ $\infty$ with server breakdowns and repairs, and the second fraction is the probability-generating function of the number of customers in the orbit given that the server is idle. 
This result can be summarized in the next theorem.

Theorem 2. The total number of customers in the system under study $(L)$ can be represented as the sum of two independent random variables: one of them is the total number of customers in the system Geo/G/1/ $\infty$ with server breakdowns and repairs $\left(L_{0}\right)$, and the other is the number of repeated customers given that the server is idle (M). That is, $L=L_{0}+M$.

As an application of the stochastic decomposition law, in the next theorem, we provide a measure of the proximity between the steady-state distributions for the standard $\mathrm{Geo} / G / 1 / \infty$ with server breakdown and repairs and the system under consideration.

Theorem 3. The following inequalities hold:

$$
\begin{aligned}
& 2 \frac{[(\bar{a}+a \bar{\theta}) v-S[(\bar{a}+a \bar{\theta}) v]]\left(a \bar{\theta}+(\bar{a}+a \bar{\theta}) \bar{v}\left[\bar{a}+a R^{\prime}(1)\right]\right)}{(\bar{a}+a \bar{\theta})[v S[(\bar{a}+a \bar{\theta}) v]+a \theta v]}[1-A(\bar{a})] \leq \\
& \leq \sum_{j=0}^{\infty}\left|P[L=j]-P\left[L_{0}=j\right]\right| \leq \\
& \leq 2 \frac{[(\bar{a}+a \bar{\theta}) v-S[(\bar{a}+a \bar{\theta}) v]]\left(a \bar{\theta}+(\bar{a}+a \bar{\theta}) \bar{v}\left[\bar{a}+a R^{\prime}(1)\right]\right)}{\bar{a}(\bar{v}+a \theta v) S[(\bar{a}+a \bar{\theta}) v]-[(\bar{a}+a \bar{\theta}) v-S[(\bar{a}+a \bar{\theta}) v]]\left(a \bar{\theta}+(\bar{a}+a \bar{\theta}) \bar{v} a R^{\prime}(1)\right)} . \\
& . \quad[1-A(\bar{a})]
\end{aligned}
$$

The proof of this theorem is omitted, but can be deduced using the steps given in [19]. Finally, let us observe that the distance $\sum_{j=0}^{\infty}\left|P[L=j]-P\left[L_{0}=j\right]\right|$ between the distributions of the random variables $L$ and $L_{0}$ decreases as $A(\bar{a})$ approaches one.

\section{Calculation of Steady-State Probabilities}

This section introduces recursive formulae to calculate the steady-state distributions of the orbit and system size.

Theorem 4. The steady-state distribution of the orbit size is given by the following recursive formulae:

$$
\begin{aligned}
\psi_{0} & =P[N=0]=\frac{a v+\bar{v} S(\bar{a} v)}{(1-\bar{a} v) S(\bar{a} v)} \cdot \pi_{0,0} \\
\psi_{k} & =P[N=k]= \\
& =\frac{\sum_{n=0}^{k-1}\left[l_{k-n}-\bar{a} A(\bar{a}) c_{k-n}\right] \psi_{n}+\left[\gamma_{k}-\bar{a} b_{k+1}-a \bar{\theta} b_{k}\right] A(\bar{a}) \pi_{0,0}}{A(\bar{a}) S(\bar{a} v)} v, k \geq 1
\end{aligned}
$$


where:

$$
\begin{aligned}
& l_{n}=\sum_{i=1}^{n} b_{i} l_{n-i}, n \geq 1 \\
& e_{0}=1-\bar{a} v-\bar{a} A(\bar{a}) R(\bar{a}) \bar{v} \\
& e_{1}=\left\{1-a \theta-\left(\bar{a}^{2} A(\bar{a}) d_{1}+[1+a \bar{\theta} A(\bar{a})] R(\bar{a})\right)\right\} \bar{v} \\
& e_{n}=\left\{(1-a \theta)\left[\frac{\bar{a}-R(\bar{a})}{\bar{a}}-\sum_{i=1}^{n-2} d i\right]-\bar{a}[1+a \bar{\theta} A(\bar{a})] d_{n-1}-\bar{a} A(\bar{a}) d_{n}\right\} \bar{v}, n \geq 2 \\
& d_{n}=\sum_{i=n}^{\infty}\left(\begin{array}{c}
n \\
i
\end{array}\right) r_{i+1} \bar{a}^{i-n} a^{n}, n \geq 1 \\
& \left.b_{n}=\sum_{i=n}^{\infty}\left(\begin{array}{c}
n-1 \\
i-1
\end{array}\right)\right) S_{i+1} v^{i-1} \bar{a}^{i-n}(a \bar{\theta})^{n-1}, n \geq 1 \\
& c_{n}=\sum_{i=n}^{\infty}\left(\begin{array}{c}
n \\
i
\end{array}\right) s_{i+1} v^{i} \bar{a}^{i-n}(a \bar{\theta})^{n}, n \geq 1 \\
& \gamma_{n}=\sum_{i=n}^{\infty}\left(\begin{array}{c}
n \\
i
\end{array}\right) S_{i+1} v^{i} \bar{a}^{i-n}(a \bar{\theta})^{n}, n \geq 1 \\
& \gamma_{0}=\frac{1-S(\bar{a} v)}{1-\bar{a} v} .
\end{aligned}
$$

Proof. Let us note that the GF $\psi(z)$ of the number of customers in the orbit satisfies the following relation:

$$
\begin{aligned}
\psi(z) G(z) & =\frac{1}{1-(\bar{a}+a \bar{\theta} z) v}[1-S[(\bar{a}+a \bar{\theta} z) v]-(\bar{a}+a \bar{\theta} z)[1- \\
& \left.\left.-\frac{S[(\bar{a}+a \bar{\theta} z) v]}{(\bar{a}+a \bar{\theta} z) v}\right]\right] A(\bar{a}) \pi_{0,0}
\end{aligned}
$$

where:

$$
\begin{aligned}
G(z) & =\bar{a} A(\bar{a}) \frac{S[(\bar{a}+a \bar{\theta} z) v]}{(\bar{a}+a \bar{\theta} z) v}-\frac{z}{1-(\bar{a}+a \bar{\theta} z) v}\left[1-\frac{S[(\bar{a}+a \bar{\theta} z) v]}{(\bar{a}+a \bar{\theta} z) v}\right]\left(\frac{k(z)}{1-z}-\right. \\
& \left.-\frac{z+\bar{a} A(\bar{a})(1-z)}{1-z}(\bar{a}+a \bar{\theta} z) \bar{v} \frac{R(\bar{a}+a z)}{\bar{a}+a z}\right)=\sum_{n=0}^{\infty} g_{n} z^{n}
\end{aligned}
$$

In order to obtain the sequence $\left\{g_{n}\right\}_{n=0}^{\infty}$, we use the properties of the generating functions and Newton's binomial (see [17]), obtaining:

$$
G(z)=A(\bar{a}) \frac{S(\bar{a} v)}{v}-\sum_{n=1}^{\infty}\left[l_{n}-\bar{a} A(\bar{a}) c_{n}\right] z^{n} .
$$

Taking into account that the development in power series of the right-hand side of (27) is:

$$
\left(\frac{a v+\bar{v} S(\bar{a} v)}{v(1-\bar{a} v}+\sum_{n=1}^{\infty}\left[\gamma_{n}-\bar{a} b_{n+1}-a \bar{\theta} b_{n}\right] z^{n}\right) A(\bar{a}) \pi_{0,0}
$$


and equating the coefficients of $z^{k}$ on both sides of (27) lead to:

$$
\begin{aligned}
\psi_{0} g_{0} & =\frac{a v+\bar{v} S(\bar{a} v)}{v(1-\bar{a} v)} A(\bar{a}) \pi_{0,0} \\
\sum_{n=0}^{k} \psi_{n} g_{k-n} & =\left[\gamma_{k}-\bar{a} b_{k+1}-a \bar{\theta} b_{k}\right] A(\bar{a}) \pi_{0,0}
\end{aligned}
$$

Since $g_{0}=\frac{A(\bar{a}) S(\bar{a} v)}{v}$ and $g_{n}=\bar{a} A(\bar{a}) c_{n}-l_{n}, n \geq 1$, Equations (25) and (26) are readily obtained.

Theorem 5. The steady-state distribution of the system size is given by the following recursive formulae:

$$
\begin{aligned}
\Phi_{0} & =P[L=0]=\pi_{0,0} \\
\Phi_{k} & =P[L=k]= \\
& =\frac{1}{A(\bar{a}) S(\bar{a} v)}\left[\sum_{n=0}^{k-1}\left[l_{k-n}-\bar{a} A(\bar{a}) c_{k-n}\right] \Phi_{n}+\left[\bar{a} \gamma_{k}+\right.\right. \\
& \left.\left.+a \gamma_{k-1}-\bar{a} b_{k+1}-a \bar{\theta} b_{k}\right] A(\bar{a}) \pi_{0,0}\right] v
\end{aligned}
$$

Proof. The GF $\Phi(z)$ of the number of customers in the system satisfies the following relation:

$$
\begin{aligned}
\Phi(z) G(z) & =\frac{1}{1-(\bar{a}+a \bar{\theta} z) v}[(\bar{a}+a z)[1-S[(\bar{a}+a \bar{\theta} z) v]]- \\
& \left.-(\bar{a}+a \bar{\theta} z)\left[1-\frac{S[(\bar{a}+a \bar{\theta} z) v]}{(\bar{a}+a \bar{\theta} z) v}\right]\right] A(\bar{a}) \pi_{0,0}
\end{aligned}
$$

where $G(z)$ and its expression in power series are given in the proof of the previous theorem.

The development in power series of the right-hand side of (30) is given by:

$$
\left[\frac{S(\bar{a} v)}{v}+\sum_{n=1}^{\infty}\left[\bar{a} \gamma_{n}+a \gamma_{n-1}-\bar{a} b_{n+1}-a \bar{\theta} b_{n}\right] z^{n}\right] A(\bar{a}) \pi_{0,0}
$$

After comparing the coefficients of $z^{k}$ on both sides in Equation (30), we obtain:

$$
\begin{aligned}
\Phi_{0} g_{0} & =\frac{S(\bar{a} v)}{v} A(\bar{a}) \pi_{0,0} \\
\sum_{n=0}^{k} \Phi_{n} g_{k-n} & =\left(\bar{a} \gamma_{k}+a \gamma_{k-1}-\bar{a} b_{k+1}-a \bar{\theta} b_{k}\right) A(\bar{a}) \pi_{0,0}, k \geq 1 .
\end{aligned}
$$

Then, taking into account the expression of the sequence $\left\{g_{n}\right\}_{n=0}^{\infty}$, we obtain Equations (28) and (29).

\section{Relation to the Continuous-Time System}

This section analyzes the link between the discrete-time system under study and its continuous counterpart. More specifically, we see the approximation of the continuoustime retrial queue with server breakdowns by the considered discrete-time system. In order to achieve this approximation, the time is slotted into small intervals of equal length, so the approximation tends to an exact value when the length of the intervals goes to zero. 
We studied the continuous-time $M / G / 1$ retrial queue with general retrial times and server breakdowns where the customers arrive in accordance with a Poisson flow with rate $\lambda$.

An arriving customer that finds the server free begins its service immediately; otherwise, with probability $\theta$ it expels from the system the customer that is currently being served and commences its service, or with complementary probability $\bar{\theta}$, it leaves the server and joins the retrial group in accordance with an FCFS discipline (that is, only the customer at the head of the orbit can access the server). Successive inter-retrial times are governed by an arbitrary probability distribution function $\Gamma(x)$ with the corresponding Laplace-Stieltjes transform $\gamma(s)$. Customer service times are identically and independently distributed random variables with a common distribution function $B(x)$ and the Laplace-Stieltjes transform $\beta(s)$.

We supposed that the server can fail after a random time with an exponential distribution with parameter $\alpha$. A server with a breakdown is sent to be repaired immediately, and the customer that was receiving service goes to the head of the orbit. Repair times follow a general distribution $U(x)$ with the Laplace-Stieltjes transform $u(s)$ and finite mean $\mu^{-1}$. It is assumed that the interarrival times, inter-retrial times, service times, server lifetime, and repair times are mutually independent.

If it is supposed that time is divided into intervals of equal length $\Delta$, the continuoustime system can be approximated by a discrete-time system, where:

$$
\begin{aligned}
a=\lambda \Delta, v=1-\alpha \Delta, a_{i}=\int_{i \Delta}^{(i+1) \Delta} d \Gamma(x), i \geq 0, s_{i} & =\int_{(i-1) \Delta}^{i \Delta} d B(x), i \geq 1, \\
r_{i} & =\int_{(i-1) \Delta}^{i \Delta} d U(x), i \geq 1
\end{aligned}
$$

and $\Delta$ must be chosen small enough so that $a$ and $v$ are probabilities.

Our objective was to prove that $\lim _{\Delta \rightarrow 0} \Phi(z)$ is the probability-generating function of the number of customers in the $M / G / 1$ retrial queueing system with general retrial times and server breakdowns. First, it is easy to prove the following equalities by applying the definition of Lebesgue integration:

$$
\begin{aligned}
\lim _{\Delta \rightarrow 0} A(\bar{a}) & =\gamma(\lambda) \\
\lim _{\Delta \rightarrow 0} S[(\bar{a}+a \bar{\theta} z) v] & =\beta[\alpha+\lambda(1-\bar{\theta} z)] \\
\lim _{\Delta \rightarrow 0} R(\bar{a}+a z) & =u[\lambda(1-z)] .
\end{aligned}
$$

The proof of these relations is omitted; the technique used can be seen in [17], Theorem 5 . 
Considering the above results, we obtain the next relation:

$$
\begin{aligned}
\lim _{\Delta \rightarrow 0} \Phi(z)= & \lim _{\Delta \rightarrow 0} \frac{A(\bar{a})(1-z)(\bar{a}+a z)(\bar{a}+a \bar{\theta} z)[(1-(\bar{a}+a z) v) S[(\bar{a}+a \bar{\theta} z) v]+a \theta v z]}{D(z)} . \\
& \cdot \pi_{0,0}= \\
& \lim _{\Delta \rightarrow 0}(A(\bar{a})(1-z)[1-\lambda \Delta(1-z)][1-\lambda \Delta(1-\bar{\theta} z)][\Delta\{\alpha+ \\
& +\lambda(1-z)-\alpha \lambda \Delta(1-z)\} S[(\bar{a}+a \bar{\theta} z) v]+\Delta \lambda \theta z[1-\alpha \Delta]]) . \\
& \cdot\{(1-\lambda \Delta) A(\bar{a})(1-z)[1-\lambda \Delta(1-z)] \Delta\{\alpha+\lambda(1-\bar{\theta} z)- \\
& -\lambda \alpha \Delta(1-\bar{\theta} z)\} S[(\bar{a}+\bar{a} \bar{\theta} z) v]-z[[1-\lambda \Delta(1-\bar{\theta} z)][1-\alpha \Delta]- \\
& -S[(\bar{a}+a \bar{\theta} z) v]][[1-\lambda \Delta(1-z)] \Delta[\alpha+\lambda \bar{\theta}(1-z)-\lambda \alpha \Delta(1-\bar{\theta} z)]- \\
& -[z+(1-\lambda \Delta) A(\bar{a})(1-z)] \alpha \Delta[1-\lambda \Delta(1-\bar{\theta} z)] R(\bar{a}+a z)]\}^{-1} \pi_{0,0} \\
= & \frac{N}{D} \pi_{0,0}^{*}
\end{aligned}
$$

where:

$$
\begin{aligned}
N & =\gamma(\lambda)(1-z)[[\alpha+\lambda(1-z)] \beta[\alpha+\lambda(1-\bar{\theta} z)]+\lambda \theta z] \\
D & =\gamma(\lambda)(1-z)[\alpha+\lambda(1-\bar{\theta} z)] \beta[\alpha+\lambda(1-\bar{\theta} z)]- \\
& -z[1-\beta[\alpha+\lambda(1-\bar{\theta} z)]][\alpha+\lambda \bar{\theta}(1-z)-[z+\gamma(\lambda)(1-z)] \alpha u[\lambda(1-z)]] \\
\pi_{0,0}^{*} & =\frac{\gamma(\lambda)(\alpha+\lambda \theta) \beta(\alpha+\lambda \theta)-[1-\beta[\alpha+\lambda \theta]]\left[\lambda \bar{\theta}+\alpha\left\{1-\gamma(\lambda)+\lambda \mu^{-} 1\right\}\right]}{[\alpha \beta(\alpha+\lambda \theta)+\lambda \theta] \gamma(\lambda)} .
\end{aligned}
$$

\section{Busy Period}

At this point, we studied an auxiliary system that is different from the original one by two facts: one is that the probability of an arrival is $a \theta$, and the other is that a customer entering the system goes directly to the server, interrupting the current customer's service, if any. As in the original system, the interrupted customer is expelled from the system, and in the case that during a service, the server's failure occurs, the customer that was in the server is placed at the head of the orbit. For this auxiliary system, we find the distribution of the busy period that will be useful to study the customers' delay in the original system.

A busy period is defined as the period starting with the arrival of a customer that finds the system empty and ending at the first service completion when the system becomes empty again, and no external arrivals take place.

Let $h_{k}, k \geq 0$, be the probability that the busy period lasts $k$ slots. Then, we have:

$$
\begin{aligned}
& h_{0}=0 \\
& h_{k}=[(1-a \theta) v]^{k-1}(1-a \theta) s_{k}+\sum_{i=1}^{k}[(1-a \theta) v]^{i-1} s_{i} a \theta h_{k-i}+ \\
+ & \sum_{i=1}^{k}[(1-a \theta) v]^{i-1} S_{i+1} a \theta h_{k-i}+ \\
+ & \sum_{i=1}^{k}[(1-a \theta) v]^{i-1} S_{i+1}(1-a \theta) \bar{v} \sum_{j=1}^{k-i} r_{j} \sum_{m=0}^{k-i-j} w_{m}^{*} h_{k-i-j-m}, k \geq 1
\end{aligned}
$$


where $w_{m}^{*}, m \geq 0$, is the probability that the customer displaced in the moment of the failure occurrence to the first place of the orbit spends there $m$ slots since the ending of the repair time till the beginning of its service.

We mention the less obvious terms of the above formula, that is the third and fourth ones, respectively:

- The arriving customer chooses a service time that lasts no less than $i+1, i=1 \ldots, k$ slots (with probability $S_{i+1}$ ). In the first $i-1$ slots, no customer arrives and no failures take place (with probability $((1-a \theta) v)^{i-1}$ ), and in the slot $i$, a new customer arrives (with probability $a \theta$ ), initiating a busy period of length $k-i$ slots (with probability $\left.h_{k-i}\right)$;

- This term differs from the previous one, that in the slot $i$, a failure takes place with probability $\bar{v}$, then after a repair time of $j, j=1, \ldots, k-i$, slots, the displaced customer to the orbit will last $m, m=0, \ldots, k-i-j$, slots, to begin its service and, then, once in the server, will open a busy period of $k-i-j-m$ slots (all with probability $\left.r_{j} w_{m}^{*} h_{k-i-j-m}\right)$.

The GF $h(x)=\sum_{k=0}^{\infty} h_{k} x^{k}$ of the BP satisfies the following equation:

$$
\begin{aligned}
h(x) & =\frac{1}{v} S[(1-a \theta) v x]+\frac{a \theta}{(1-a \theta) v} S[[(1-a \theta) v x] h(x)+ \\
& +\frac{a \theta}{(1-a \theta) v} \cdot \frac{(1-a \theta) v x-S[(1-a \theta) v x]}{1-(1-a \theta) v x} h(x)+ \\
& +\frac{\bar{v}}{v} \cdot \frac{(1-a \theta) v x-S[(1-a \theta) v x]}{1-(1-a \theta) v x} \cdot R(x) w^{*}(x) h(x),
\end{aligned}
$$

where $w^{*}(x)$ is the GF associated with the distribution $\left\{w_{m}^{*}, m \geq 0\right\}$.

The above equation can be written in the form:

$$
\begin{aligned}
& {[a \theta[1-(1-a \theta) v x] S[(1-a \theta) v x]+a \theta[(1-a \theta) v x-S[(1-a \theta) v x]]+} \\
+ & (1-a \theta) \bar{v}[(1-a \theta) v x-S[(1-a \theta) v x]] R(x) w^{*}(x)- \\
- & (1-a \theta) v[1-(1-a \theta) v x]]] h(x)+ \\
+ & (1-a \theta)[1-(1-a \theta) v x] S[(1-a \theta) v x]=0 .
\end{aligned}
$$

In order to obtain the GF $w^{*}(x)$, we need to know first the probabilities $w_{k}, k \geq 0$, that the period of time, that the customer placed at the head of the orbit remains there since the end of a BP till the beginning of its service, lasts exactly $k$ slots.

The probabilities $w_{k}, k \geq 0$, are governed by the following recursive formulae:

$$
\begin{aligned}
& w_{0}=a_{0} \\
& w_{k}=(1-a \theta)^{k} a_{k}+\left(1-\delta_{1, k}\right) a \theta \sum_{l=1}^{k-1}(1-a \theta)^{l-1} A_{l} \sum_{i=1}^{k-l} h_{i} w_{k-l-i}, k \geq 1,
\end{aligned}
$$

where $A_{l}=\sum_{k=l}^{\infty} a_{k}$ is the probability that, until the slot $l$, no retrial has taken place.

Let us explain the above formula. Let us call 0 the slot in which a BP finishes. At this moment, the customer at the head of the orbit will start its service immediately with probability $a_{0}$ (we remind that no customer arrives in Slot 0 , since in this slot, a BP has finished). Now. we consider the equation for $k \geq 1$. The customer at the head of the orbit will wait there $k$ slots, $k \geq 1$, from the completion of a BP till the beginning of its service if: 
(a) In the first $k$ slots, no customers arrive, and in the slot $k$, a retrial occurs (with probability $\left.(1-a \theta)^{k} a_{k}\right)$ or;

(b) Before the slot $l, 1 \leq l \leq k-1$, no customer arrives at the system and no retrial occurs (with probability $\left.(1-a \theta)^{l-1} A_{l}\right)$, and in the slot $l$, a new customer arrives at the system, opening a BP with length $i(i=1, \ldots, k-l)$; upon the completion of this $\mathrm{BP}$, the customer placed at the head of the orbit will wait there till the beginning of its service $k-l-i$ slots (all with probability $a \theta h_{i} w_{k-l-i}$ ).

The GF of the distribution $w(x)=\sum_{k=0}^{\infty} w_{k} x^{k}$ is given by:

$$
w(x)=\frac{[1-(1-a \theta) x] A[(1-a \theta) x]}{1-(1-a \theta) x-a \theta x h(x)[1-A[(1-a \theta) x]]},
$$

with mean

$$
\bar{w}=w^{\prime}(1)=\frac{(1+a \theta \bar{h})[1-A(1-a \theta)]}{a \theta A(1-a \theta)},
$$

where $\bar{h}=\mathrm{h}^{\prime}(1)$.

Now, we are in a position to write down the expression of the GF $w^{*}(x)$, which has the following form:

$$
w^{*}(x)=(1-a \theta+a \theta h(x)) w(x) .
$$

with mean:

$$
\begin{aligned}
\bar{w}^{*} & =\left(w^{*}\right)^{\prime}(1)=a \theta \bar{h}+\bar{w}= \\
& =\frac{[1-A(1-a \theta)]+a \theta \bar{h}[1-(1-a \theta) A(1-a \theta)]}{a \theta A(1-a \theta)} .
\end{aligned}
$$

Inserting the expression of $w^{*}(x)$ in (32), we obtain:

$$
\alpha(x) h^{2}(x)+\beta(x) h(x)+\gamma(x)=0,
$$

where:

$$
\begin{aligned}
\alpha(x) & =a \theta[-a \theta x[1-A[(1-a \theta) x]][1-(1-a \theta) v x] S[(1-a \theta) v x]- \\
- & a \theta x[1-A[(1-a \theta) x]][(1-a \theta) v x-S[(1-a \theta) v x]]+ \\
+ & {[1-(1-a \theta) x] A[(1-a \theta) x](1-a \theta) \bar{v}[(1-a \theta) v x-} \\
- & S[(1-a \theta) v x]] R(x)+[1-A[(1-a \theta) x]] . \\
& (1-a \theta) v x[1-(1-a \theta) v x]]
\end{aligned}
$$




$$
\begin{aligned}
\beta(x)= & a \theta[1-(1-a \theta) v x][1-(1-a \theta) x] S[(1-a \theta) v x]+ \\
+ & a \theta[1-(1-a \theta) x][(1-a \theta) v x-S[(1-a \theta) v x]]+ \\
+ & (1-a \theta)^{2} \bar{v}[1-(1-a \theta) x] A[(1-a \theta) x][(1-a \theta) v x- \\
- & S[(1-a \theta) v x]] R(x)-(1-a \theta) v[1-(1-a \theta) x] . \\
\cdot & {[1-(1-a \theta) v x]-a \theta(1-a \theta)[1-A[(1-a \theta) x]][1-(1-a \theta) v x] . } \\
\cdot & S[(1-a \theta) v x]
\end{aligned}
$$

and:

$$
\gamma(x)=(1-a \theta)[1-(1-a \theta) x][1-(1-a \theta) v x] S[(1-a \theta) v x] .
$$

Equation (38) shows that the GF $h=h(x)$ satisfies the quadratic equation:

$$
f(h)=0
$$

where:

$$
f(h)=\alpha(x) h^{2}+\beta(x) h+\gamma(x) .
$$

Let us note that for any fix, $x \in(0,1)$ is:

$$
\begin{aligned}
& \alpha(x)>0 \\
& f(0)=\gamma(x)>0 \\
& f(1)<(1-a \theta) v(x-1)[1-S[(1-a \theta) v x]] .
\end{aligned}
$$

The above relations show that for any $x \in(0,1)$, Formula (35) has two solutions, $h(x)$ and $h^{*}(x)$, satisfying the inequalities $0<h(x)<1<h^{*}(x)$ and given by:

$$
\begin{aligned}
h(x) & =\frac{-\beta(x)-\left[\beta^{2}(x) 4 \alpha(x) \gamma(x)\right]^{1 / 2}}{2 \alpha(x)} \\
h^{*}(x) & =\frac{-\beta(x)+\left[\beta^{2}(x) 4 \alpha(x) \gamma(x)\right]^{1 / 2}}{2 \alpha(x)} .
\end{aligned}
$$

For $x=1$, it is $f(1)=0$, which means that at least one of the two solutions $h(x)$ or $h^{*}(x)$ takes the value one for $x=1$. Let us observe that the inequality $h^{*}(1)>1$ holds if and only if:

$$
\begin{aligned}
\sqrt{\beta(1)^{2}-4 \alpha(1) \gamma(1)}> & 2 \alpha(1)+\beta(1)= \\
= & a \theta[-(1-a \theta)(\bar{v}+a \theta v) A(1-a \theta) S[(1-a \theta) v]+ \\
+ & {[(1-a \theta) v-S[(1-a \theta) v]] . } \\
& (1-a \theta) \bar{v}[1-(1-a \theta) A(1-a \theta)]]
\end{aligned}
$$

However, if the stability condition (24) is fulfilled, the right-hand side of the above inequality is negative and, as a consequence, $h^{*}(1)>1$ and $h(1)=1$; therefore, the GF of the busy period is $h(x)$. 
The mean length of a BP is given by:

$$
\bar{h}=h^{\prime}(1)=\frac{N}{D}
$$

where:

$$
\begin{aligned}
N= & (1-a \theta) v[1-S[(1-a \theta) v]]+[(1-a \theta) v-S[(1-a \theta) v]] \\
\cdot & {\left[(1-a \theta) \bar{v}[1-A(1-a \theta)]+R^{\prime}(1)\right.} \\
D= & a \theta\{(1-a \theta)(\bar{v}+a \theta v) A(1-a \theta) S[(1-a \theta) v]-[(1-a \theta) v-S[(1-a \theta) v]] . \\
\cdot & ((1-a \theta) \bar{v}[1-(1-a \theta) A(1-a \theta)]\}
\end{aligned}
$$

At this stage, we consider the GF $h(x ; m)$ of the BP that starts with a customer with $m$ remaining slots to finish its service. This GF will be useful in the study of the customers' delay.

The GF $h(x, m)$ is given by:

$$
\begin{aligned}
h(x ; m) & =\frac{[(1-a \theta) v x]^{m}}{(1-a \theta) v}[1-a \theta+a \theta h(x)]+ \\
& +x \frac{1-[(1-a \theta) v x]^{m-1}}{1-(1-a \theta) v x}\left[a \theta h(x)+(1-a \theta) \bar{v} R(x) w^{*}(x) h(x)\right], m \geq 1
\end{aligned}
$$

Let us explain the above formula:

If after the first $m-1$ slots, no customer arrives at the system and no failure has occurred (with probability $[(1-a \theta) v]^{m-1}$ ), and in the slot $m$, either a new customer does not arrive, then the BP ends with probability $1-a \theta$, or another customer arrives, with probability $a \theta$, then a new BP is opened with GF $h(x)$.

If after $k-1$ slots, $k=1, \ldots, m-1$, a new customer does not arrive and no failures have taken place (with probability $[(1-a \theta) v]^{k-1}$ ) and in the slot $k$, a new customer arrives (with probability $a \theta$ ) opening a BP with GF $h(x)$ or no customer arrives and a failure occurs (with probability $(1-a \theta) \bar{v}$ ), then a period of repair time begins with GF $R(x)$; once this repair time has finished, the customer displaced to the orbit in the moment of the occurrence of the failure will wait there till the beginning of its service time with GF $w^{*}(x)$, after which this customer will open a BP with GF $h(x)$. Summing over $k$ from one to $m-1$, the formula of $h(x ; m)$ is obtained.

Let us note that the expression of $h(x ; m)$ can be written as follows:

$$
\begin{aligned}
h(x ; m) & =\frac{1}{v[1-(1-a \theta) v x]}\left\{[(1-a \theta) v x]^{m}[1-v x(1-a \theta+a \theta h(x))-\right. \\
& \left.\left.-\bar{v} R(x) w^{*}(x) h(x)\right]+v x\left[a \theta h(x)+(1-a \theta) \bar{v} R(x) w^{*}(x) h(x)\right]\right\}
\end{aligned}
$$

\section{Sojourn Times}

\subsection{Sojourn Time of a Customer in the Server}

This section is dedicated to obtaining the distribution of the time that a customer spends in the server. As the service of a customer can be interrupted, in case a failure in the server takes place, the sojourn time of a customer in the server may consist of separate time intervals. 
Let us denote by $b_{k}$ the probability that the sojourn time of a customer in the server (taking into account possible disruptions) lasts exactly $k$ slots. The distribution $\left\{b_{k}, k \geq 0\right\}$ is governed by the following recursive formulae:

$$
\begin{aligned}
b_{0} & =0 \\
b_{k} & =[(\bar{a}+a \bar{\theta}) v]^{k-1} s_{k}+[(\bar{a}+a \bar{\theta}) v]^{k-1} S_{k+1} a \theta+ \\
& +\sum_{i=1}^{k}[(\bar{a}+a \bar{\theta}) v]^{i-1}(\bar{a}+a \bar{\theta}) \bar{v} S_{i+1} b_{k-i}, k \geq 1
\end{aligned}
$$

The GF $b(x)=\sum_{k=0}^{\infty} b_{k} x^{k}$ is given by:

$$
\begin{aligned}
b(x) & =\frac{1}{(\bar{a}+a \bar{\theta}) v} S[(\bar{a}+a \bar{\theta}) v x]+ \\
& +\frac{a \theta}{(\bar{a}+a \bar{\theta}) v} \cdot \frac{(\bar{a}+a \bar{\theta}) v x-S[(\bar{a}+a \bar{\theta}) v x]}{1-(\bar{a}+a \bar{\theta}) v x}+ \\
& +\frac{\bar{v}}{v} \cdot \frac{(\bar{a}+a \bar{\theta}) v x-S[(\bar{a}+a \bar{\theta}) v x]}{1-(\bar{a}+a \bar{\theta}) v x} b(x)
\end{aligned}
$$

that is,

$$
b(x)=\frac{[1-v x] S[\bar{a}+a \bar{\theta}) v x]+a \theta v x}{[1-(\bar{a}+a \bar{\theta}) x] v+\bar{v} S[(\bar{a}+a \bar{\theta}) v x]},
$$

and the mean sojourn time of a customer in the server is:

$$
\bar{b}=b^{\prime}(1)=\frac{v[1-S[(\bar{a}+a \bar{\theta}) v]]}{a \theta v+\bar{v} S[(\bar{a}+a \bar{\theta}) v]} .
$$

\subsection{Sojourn Time of a Customer in the Orbit}

The stationary distribution of the waiting time that a customer spends in the orbit until the beginning of its service has the following GF:

$$
\begin{aligned}
W(x) & =\pi_{0,0}+\sum_{i=1}^{\infty} \sum_{k=1}^{\infty} \pi_{0,1, k}+\sum_{i=1}^{\infty} \sum_{k=0}^{\infty} \pi_{1,1, k}+\sum_{k=0}^{\infty} \pi_{2,1, k}+ \\
& +\theta \sum_{i=1}^{\infty} \sum_{k=0}^{\infty} \pi_{1, i+1, k}+ \\
& +\bar{\theta} w(x) \sum_{i=1}^{\infty} \sum_{k=0}^{\infty} \pi_{1, i+1, k} h(x ; i)(h(x) w(x))^{k}+ \\
& +w^{*}(x) \sum_{i=1}^{\infty} \sum_{k=1}^{\infty} \pi_{2, i+1, k} x^{i}(h(x) w(x))^{k}
\end{aligned}
$$


Using (40) and the GF's introduced in Section 3 the above formula becomes:

$$
\begin{aligned}
W(x) & =\pi_{0,0}+\varphi_{0}(1,1)+\bar{\theta} \varphi_{1,1}(1)+\varphi_{2,1}(1)+\theta \varphi_{1}(1,1)+ \\
& +\bar{\theta} w(x)\left\{F_{1}(x) \frac{1}{(1-a \theta) v x} \varphi_{1}[(1-a \theta) v x, h(x) w(x)]+\right. \\
& \left.+F_{2}(x) \varphi_{1}[1, h(x) w(x)]-\frac{1-\bar{v} R(x) w^{*}(x) h(x)}{v} \varphi_{1,1}(h(x) w(x))\right\}+ \\
& +w^{*}(x)\left[\frac{1}{x} \varphi_{2}(x, h(x) w(x))-\varphi_{2,1}(h(x) w(x))\right]
\end{aligned}
$$

where:

$$
\begin{aligned}
& F_{1}(x)=\frac{1-v x(1-a \theta+a \theta h(x))-\bar{v} R(x) w^{*}(x) h(x)}{v[1-(1-a \theta) v x]}, F_{1}(1)=0 \\
& F_{2}(x)=\frac{x\left[a \theta h(x)+(1-a \theta) \bar{v} R(x) w^{*}(x) h(x)\right]}{1-(1-a \theta) v x}, F_{2}(1)=1
\end{aligned}
$$

and its expected value is:

$$
\begin{aligned}
\bar{W} & =W^{\prime}(1)= \\
& =\bar{\theta}\left\{\bar{w}\left[\varphi_{1}(1,1)-\varphi_{1,1}(1)\right]+\right. \\
& +F_{1}^{\prime}(1) \frac{1}{(1-a \theta) v} \varphi_{1}((1-a \theta) v, 1)+F_{2}^{\prime}(1) \varphi_{1}(1,1)+\varphi_{1}^{\prime}(1, h(x) w(x))_{x=1}+ \\
& \left.+\frac{1}{v}\left[\bar{v}\left(R^{\prime}(1)+\bar{w}^{*}+\bar{h}\right)\right] \varphi_{1,1}(1)-\varphi_{1,1}^{\prime}(h(x) w(x))_{x=1}\right\}+ \\
& +\bar{w}^{*}\left[\varphi_{2}(1,1)-\varphi_{2,1}(1)\right]+\left(\frac{\varphi_{2}^{\prime}(x, h(x) w(x))}{x}\right)_{x=1}-\varphi_{2,1}^{\prime}(h(x) w(x))_{x=1}
\end{aligned}
$$

where:

$$
\begin{aligned}
F_{1}^{\prime}(1) & =\frac{v(1+a \theta \bar{h})+\bar{v}\left(R^{\prime}(1)+\bar{w}^{*}+\bar{h}\right)}{v(\bar{v}+a \theta v)} \\
F_{2}^{\prime}(1) & =\frac{1+a \theta \bar{h}+(1-a \theta) \bar{v}\left(R^{\prime}(1)+\bar{w}^{*}+\bar{h}\right)}{\bar{v}+a \theta v} . \\
\frac{\varphi_{1}[(1-a \theta) v, 1]}{(1-a \theta) v} & =\frac{S^{\prime}[(1-a \theta) v] a v(\bar{v}+a \theta v)}{a \theta v+\bar{v} S[(1-a \theta) v]}
\end{aligned}
$$




$$
\begin{aligned}
& \varphi_{1,1}(h(x) w(x))_{x=1}^{\prime}= \\
& =\frac{(\bar{v}+a \theta v) S[(\bar{a}+a \bar{\theta}) v] D^{\prime \prime}(1)-2 a\left\{(\bar{v}+a \theta v-\bar{\theta} \bar{v}) S[(\bar{a}+a \bar{\theta}) v] S[(\bar{a}+a \bar{\theta}) v]+\bar{\theta} v(\bar{v}+a \theta v) S^{\prime}[(\bar{a}+a \bar{\theta}) v]\right\} D^{\prime}(1)}{-2 D^{\prime}(1)(\bar{a}+a \bar{\theta})[a \theta v+\bar{v} S[(\bar{a}+a \bar{\theta}) v]]} \\
& \varphi_{2,1}(h(x) w(x))_{x=1}^{\prime}= \\
& =\frac{1}{-2 D^{\prime}(1)(\bar{a}+a \bar{\theta})[a \theta v+\bar{v} S[(\bar{a}+a \bar{\theta}) v]]}\left[(\bar{a}+a \bar{\theta})[(\bar{a}+a \bar{\theta}) v-S[(\bar{a}+a \bar{\theta}) v]] D^{\prime \prime}(1)-\right. \\
& \left.-2\left\{[(\bar{a}+a \bar{\theta}) v-S[(\bar{a}+a \bar{\theta}) v]]\left[a \bar{\theta}+(\bar{a}+a \bar{\theta})\left[1+a R^{\prime}(1)\right]\right]+a \bar{\theta} v(\bar{a}+a \bar{\theta})\left[1-S^{\prime}[(\bar{a}+a \bar{\theta}) v]\right]\right\} D^{\prime}(1)(\bar{h}+\bar{w}) a \bar{v}\right] . \\
& \varphi_{1}[1, h(x) w(x)]_{x=1}^{\prime}= \\
& =\frac{[1-S[(\bar{a}+a \bar{\theta}) v]](\bar{a}+a \bar{\theta}) v D^{\prime \prime}(1)-2 a\left\{[1-S[(\bar{a}+a \bar{\theta}) v]](\bar{\theta}+(\bar{a}+a \bar{\theta}))(\bar{a}+a \bar{\theta}) \bar{\theta} v S^{\prime}[(\bar{a}+a \bar{\theta}) v]\right\} D^{\prime}(1)}{-2 D^{\prime}(1)(\bar{a}+a \bar{\theta})[a \theta v+\bar{v} S[(\bar{a}+a \bar{\theta}) v]]} . \\
& (\bar{h}+\bar{w}) a v \\
& {\left[\frac{\varphi_{2}(x, h(x) w(x))}{x}\right]_{x=1}^{\prime}=\frac{a \bar{v}}{2(\bar{a}+a \bar{\theta})[a \theta v+\bar{v} S[(\bar{a}+a \bar{\theta}) v]]} .} \\
& \text { - } \quad R^{\prime \prime}(1)[1+a(\bar{h}+\bar{w})](\bar{a}+a \bar{\theta})[(\bar{a}+a \bar{\theta}) v-S[(\bar{a}+a \bar{\theta}) v]]+ \\
& +\quad R^{\prime}(1) \frac{1}{-2 D^{\prime}(1)}\left[( \overline { a } + a \overline { \theta } ) \left[(\bar{a}+a \bar{\theta}) v-S[(\bar{a}+a \bar{\theta}) v] D^{\prime \prime}(1)-2 a[(\bar{a}+a \bar{\theta}) v-\right.\right. \\
& \left.-\quad S[(\bar{a}+a \bar{\theta}) v]](\bar{\theta}+\bar{a}+a \bar{\theta})+a \bar{\theta} v(\bar{a}+a \bar{\theta}) v\left[1-S^{\prime}[(\bar{a}+a \bar{\theta}) v]\right] D^{\prime}(1)\right]
\end{aligned}
$$

\subsection{Sojourn Time of a Customer in the System}

We first find the distribution of the period of time that a customer spends in the system since its service begins until the moment of its departure. Let $g_{k}$ be the probability that this period of time lasts exactly $k$ slots. Then, we have:

$$
\begin{aligned}
g_{0} & =0 \\
g_{k} & =[(\bar{a}+a \bar{\theta}) v]^{k-1} s_{k}+[(\bar{a}+a \bar{\theta}) v]^{k-1} a \theta S_{k+1}+ \\
& +\sum_{i=1}^{k}[(\bar{a}+a \bar{\theta}) v]^{i-1}(\bar{a}+a \bar{\theta}) \bar{v} S_{i+1} \sum_{j=1}^{k-i} r_{j} \sum_{l=1}^{k-i-j} w_{l}^{*} g_{k-i-j-l}, k \geq 1 .
\end{aligned}
$$

The GF $g(x)=\sum_{k=0}^{\infty} g_{k} x^{k}$ is given by:

$$
\begin{aligned}
g(x) & =\frac{1}{(\bar{a}+a \bar{\theta}) v} S[(\bar{a}+a \bar{\theta}) v x]+\frac{a \theta}{(\bar{a}+a \bar{\theta}) v} \cdot \frac{[(\bar{a}+a \bar{\theta}) v x-S[(\bar{a}+a \bar{\theta}) v x]}{1-(\bar{a}+a \bar{\theta}) v x}+ \\
& +\frac{\bar{v}}{v} \cdot \frac{(\bar{a}+a \bar{\theta}) v x-S[(\bar{a}+a \bar{\theta}) v x]}{1-(\bar{a}+a \bar{\theta}) v x} R(x) w^{*}(x) g(x)
\end{aligned}
$$

that is:

$$
g(x)=\frac{a \theta v x+(1-v x) S[(\bar{a}+a \bar{\theta}) v x]}{v[1-(\bar{a}+a \bar{\theta}) v x]-\bar{v}[(\bar{a}+a \bar{\theta}) v x-S[(\bar{a}+a \bar{\theta}) v x]] R(x) w^{*}(x)} .
$$


and its corresponding mean is:

$$
\bar{g}=g^{\prime}(1)=\frac{v[1-S[(\bar{a}+a \bar{\theta}) v]]+\bar{v}[(\bar{a}+a \bar{\theta}) v-S[(\bar{a}+a \bar{\theta}) v]]\left(R^{\prime}(1)+\bar{w}^{*}\right)}{a \theta v+\bar{v} S[(\bar{a}+a \bar{\theta}) v]} .
$$

Obviously, if $v=1$, then $g(x)=b(x)$.

The GF $v(x)$ of the stationary distribution of the sojourn time of a customer in the system is:

$$
v(x)=W(x) g(x)
$$

with mean:

$$
\bar{v}=v^{\prime}(1)=\bar{W}+\bar{g} .
$$

The mean total time $\bar{W}_{T}$ that a customer spends in the orbit is given by:

$$
\bar{W}_{T}=\bar{W}+\bar{g}-\bar{b} .
$$

\section{Numerical Results}

This section presents some numerical results that illustrate the effect of the parameters on several performance characteristics of the system. Throughout this section, it was assumed that the service and repair times take exactly two slots and that the retrial times are governed by a geometrical distribution with GF $A(x)=\frac{1-r}{1-r x}$.

In Figure 1, the probability that the system is empty is plotted versus the parameter $v$. Three curves corresponding to $\theta=0,0.5,1$ are presented. As expected, $\pi_{0,0}$ increases with increasing values of $v$ and $\theta$.

Figure 2 illustrates the behavior of $E[N]$ against the parameter $v$. As intuition tell us, $E[N]$ decreases with increasing values of $v$ and $\theta$. When $v$ approaches the stability condition, the mean orbit size tends to infinity due the system becoming unstable.

In Figure 3, the stability abscissa $v^{*}$, that is the number $v^{*}$ for which for all $v \in\left(v^{*}, 1\right]$ the system is stable, is plotted against the mean repair time. The values of $v^{*}$ increase with increasing values of $R^{\prime}(1)$ and decreasing values of $\theta$, as expected, and when $R^{\prime}(1)$ tends to infinity, $v^{*}$ tends to one.

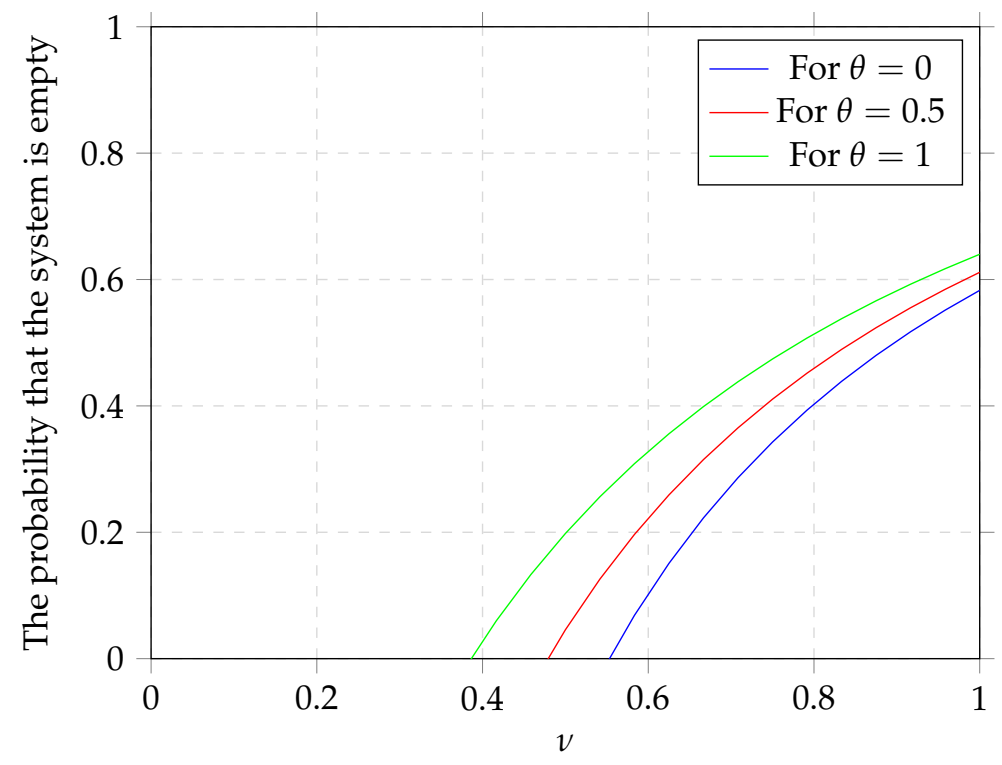

Figure 1. Probability of an empty system against $v$. 


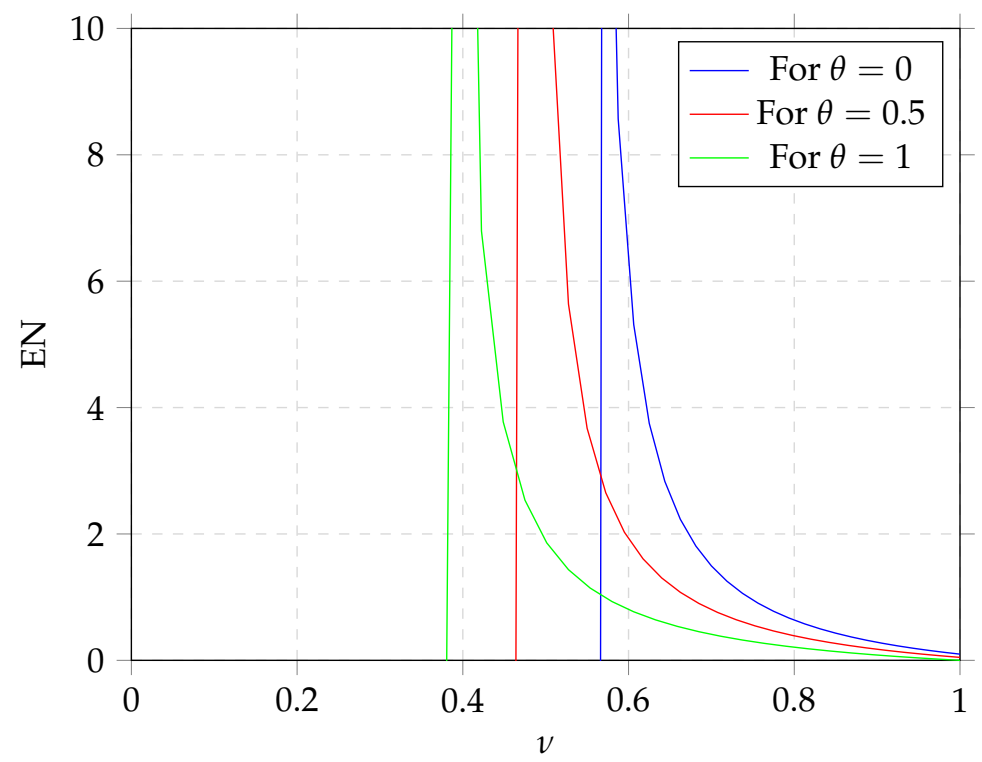

Figure 2. $E N$ against $\nu$.

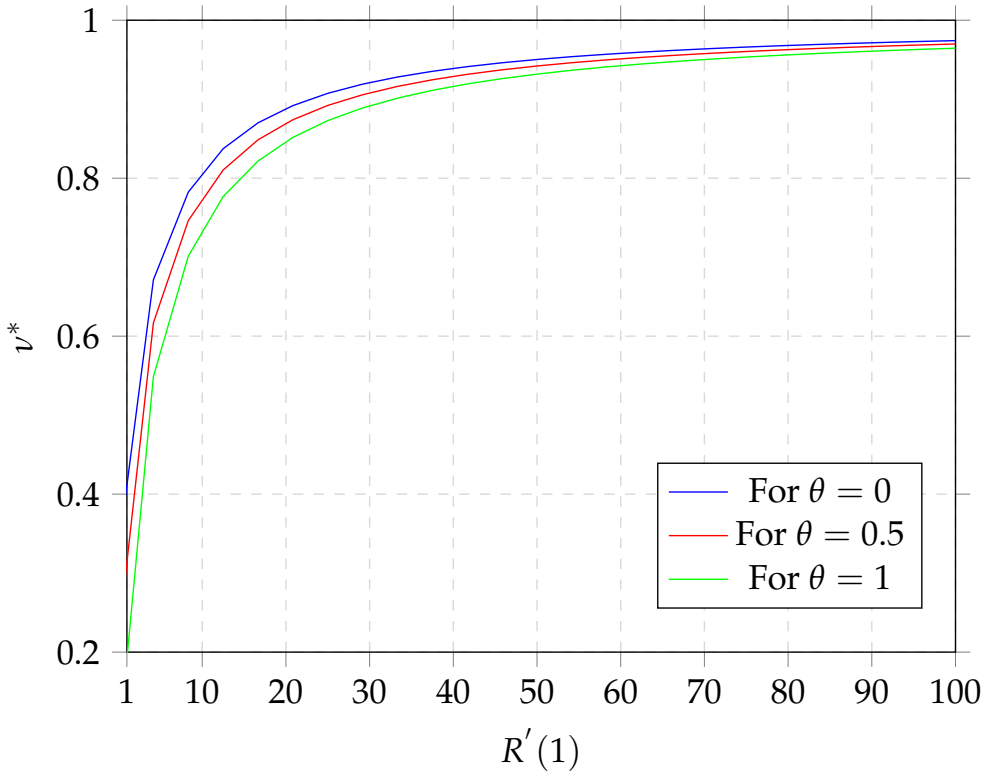

Figure 3 . The stability abscissa against the mean repair time.

The graphics tell us that for any $\theta \in[0,1]$ and any value of the mean repair time, there are always values of $v$ for which the system is stable, specifically those belonging to the interval $\left(v^{*}, 1\right]$, which is never empty.

An important feature of this paper is the recursion scheme provided by Theorems 4 and 5 , whose formulae where implemented in Tables 1 and 2. 
Table 1. The stationary distribution of the orbit size when $a=0.2, \theta=1, r=0.3$.

\begin{tabular}{ccccc}
\hline $\boldsymbol{\psi}_{\boldsymbol{k}}$ & $\boldsymbol{v}=\mathbf{0 . 4}$ & $\boldsymbol{v}=\mathbf{0 . 6}$ & $\boldsymbol{v}=\mathbf{0 . 8}$ & $\boldsymbol{v} \mathbf{\mathbf { 1 }}$ \\
\hline$k=0$ & 0.1374995 & 0.6166664 & 0.8562507 & 1 \\
$k=1$ & 0.1057027 & 0.2106944 & 0.1097071 & 0 \\
$k=2$ & 0.0941494 & 0.0976813 & 0.027435 & 0 \\
$k=3$ & 0.0822 & 0.0421533 & 0.0052292 & 0 \\
$k=4$ & 0.072084 & 0.0184723 & 0.0010985 & 0 \\
$k=5$ & 0.0631288 & 0.0080676 & 0.0002224 & 0 \\
$k=6$ & 0.055288 & 0.003526 & 0.0000455 & 0 \\
$k=7$ & 0.0484209 & 0.0015408 & 0.0000055 & 0 \\
$k=8$ & 0.0424067 & 0.0006733 & 0.0000014 & 0 \\
$k=9$ & 0.0371401 & 0.0002941 & 0.0000001 & 0 \\
$k=10$ & 0.032527 & 0.0001284 & $\approx 0$ & 0 \\
\hline
\end{tabular}

Table 2. The stationary distribution of the system size when $a=0.2, \theta=1, r=0.3$.

\begin{tabular}{ccccc}
\hline $\boldsymbol{\Phi}_{\boldsymbol{k}}$ & $\boldsymbol{v}=\mathbf{0 . 4}$ & $\boldsymbol{v}=\mathbf{0 . 6}$ & $\boldsymbol{v}=\mathbf{0 . 8}$ & $\boldsymbol{v}=\mathbf{1}$ \\
\hline$k=0$ & 0.0676921 & 0.3482352 & 0.5219052 & 0.64 \\
$k=1$ & 0.1218458 & 0.3874122 & 0.4012145 & 0.36 \\
$k=2$ & 0.100015 & 0.1468754 & 0.0595602 & 0 \\
$k=3$ & 0.088395 & 0.0663243 & 0.0134 & 0 \\
$k=4$ & 0.0772642 & 0.0287805 & 0.0027115 & 0 \\
$k=5$ & 0.0676758 & 0.0125967 & 0.0005567 & 0 \\
$k=6$ & 0.0592692 & 0.0055029 & 0.0001136 & 0 \\
$k=7$ & 0.0519077 & 0.0024049 & 0.0000231 & 0 \\
$k=8$ & 0.0454475 & 0.0010508 & 0.0000046 & 0 \\
$k=9$ & 0.039804 & 0.0004592 & 0.0000008 & 0 \\
$k=10$ & 0.0348599 & 0.0002005 & $\approx 0$ & 0 \\
\hline
\end{tabular}

\section{Conclusions and Research Results}

This paper analyzed a discrete-time retrial queue with the server subject to breakdowns and repairs is analyzed. Customers arriving at the system can decide to follow an LCFS-PR discipline or to join the orbit. We carried out an extensive study of the model and obtained the distribution of the number of customers in the orbit and in the system using a generating function approach.

An important feature of this paper is the recursive algorithm provided by Theorems 4 and 5 used to compute the steady-state probabilities of the number of customers in the orbit and in the system. We provided the stochastic decomposition law and application bounds for the proximity between the steady-state distribution of the system under consideration and the corresponding system without retrials.

We showed that the continuous-time $M / G / 1$ retrial queue with the server subject to breakdowns and repairs can be approximated by the corresponding discrete-time system. We analyzed the busy period of an auxiliary system useful in the study of the customers' delay.

A new and important research contribution to the theory of queues with general retrial times is the complete and thorough study carried out in this paper of the sojourn time distribution of a customer in the orbit and in the system. Finally, numerical examples to illustrate the influence of the parameters on several performance characteristics were given.

Author Contributions: Conceptualization, I.A. and J.L.G.G.; Investigation, I.A. and J.L.G.G.; Methodology, I.A. and J.L.G.G.; Writing_-original draft, I.A. All authors have read and agreed to the published version of the manuscript. 
Funding: This research was partially funded by the Junta de Andalucía Project UMA2018-FEDERJA001 (European Regional Development Funds).

Institutional Review Board Statement: Not applicable.

Informed Consent Statement: Not applicable.

Data Availability Statement: Not applicable.

Acknowledgments: We thank the anonymous reviewers for their suggestions and comments which have improved the quality of the paper.

Conflicts of Interest: The authors declare no conflict of interest.

\section{References}

1. Artalejo, J. G-networks: A versatile approach for work removal in queueing networks. Eur. J. Oper. Res. 2000, 126, 233-249. [CrossRef]

2. Gelenbe, E.; Label, A. G-networks with multiple classes of signals and positive customers. Eur. J. Oper. Res. 1998, 108, 293-305. [CrossRef]

3. Pechinkin, A.; Shorgin, S. The discrete-time queueing system with inversive service order and probabilistic priority. In Proceedings of the 3rd International Conference on Performance Evaluation Methodologies and Tools, Athens, Greece, 20-24 October 2008; Volume 20.

4. Krishnamoorthy, A.; Pramod, P.; Deepak, T. On a queue with interruptions and repeat or resumption of service. Nonlinear Anal. Theory, Methods Appl. 2009, 71, 1673-1683. [CrossRef]

5. Atencia, I.; Moreno, P. A discrete-time Geo/Geo/1 queue with negative customers and disasters. Comput. Oper. Res. 2004, 31, 1537-1548. [CrossRef]

6. Atencia, I.; Moreno, P. A single-server G-queue in discrete-time with geometrical arrival and service process. Perform. Eval. 2005, 59, 85-97. [CrossRef]

7. Chao, X.; Miyazawa, M.; Pinedo, M. Queueing Networks: Customers, Signals and Product form Solutions; John Wiley and Sons: Hoboken, NJ, USA, 1999.

8. Harrison, P.G.; Patel, N.M.; Pitel, E. Reliability modelling using G-queues. Eur. J. Oper. Res. 2000, 126, 273-287. [CrossRef]

9. Park, H.M.; Yang, W.S.; Chae, K.C. The Geo/G/1 queue with negative customers and disasters. Stoch. Model. 2009, 25, 673-688. [CrossRef]

10. Artalejo, R.; Gómez-Corral, A. Retrial Queueing Systems; Springer: Berlin/Heidelberg, Germany, 2008.

11. Alfa, A. Queueing Theory for Telecommunications: Discrete Time Modelling of a Single Node System; Springer: Boston, MA, USA, 2010.

12. Haghighi, A.; Mishev, D. Delayed and Network Queues; John Wiley \& Sons: Chichester, West Sussex, UK, 2016.

13. Fuhrmann, S.W.; Cooper, R.B. Stochastic decomposition in the $M / G / 1$ queue with generalized vacations. Oper. Res. 1985, 33, 1117-1129. [CrossRef]

14. Takagi, H. Queueing Analysis: A Foundation of Performance Evaluation. Discrete-Time Systems; North-Holland: Amsterdam, The Netherlands, 1993.

15. Tian, N.; Zhang, Z. System delay versus system content for discrete-time queueing systems subject to server interruptions. Discret.-Time GI / Geo/1 Queue Mult. Vacat. 2002, 40, 283-294.

16. Atencia, I.; Moreno, P. A discrete-time Geo/Geo/1 retrial queue with general retrial times. Queueing Syst. 2004, 48, 5-21. [CrossRef]

17. Yang, T.; Li, H. On the steady-state queue size distribution of the discrete-time Geo/G/1 queue with repeated customers. Queueing Syst. 1995, 21, 199-215. [CrossRef]

18. Osawa, T.H.; Fujisawa, T. $G^{[X]} / G / 1$ retrial queue with non-preemptive priority. Asia-Pacific J. Oper. Res. 1999, 16, $215-234$.

19. Artalejo, J.; Falin, G. Stochastic decomposition for retrial queues. Top 1994, 2, 329-342. [CrossRef] 